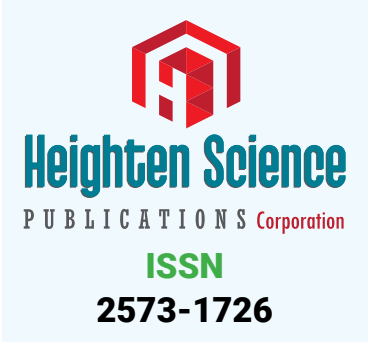

*Address for Correspondence: Zabchi Nor Eddine, Physical Education and Sport Activities Programmes Evaluation Laboratory, Physical Education and Sports Institute - University Mostganem- Algeria, Email: norzabchi7@yahoo.fr

Submitted: 28 December 2018

Approved: 28 January 2019

Published: 29 January 2019

Copyright: (c) 2019 Omar D, et al. This is an open access article distributed under the Creative Commons Attribution License, which permits unrestricted use, distribution, and reproduction in any medium, provided the original work is properly cited

Keywords: Rehabilitation program; Functional parameters; Aqua-aerobic exercises; Intensity and severity of asthma attacks
Research Article

\section{A proposed Habilitation program to improve some functional parameters using Aqua-aerobic Exercises to reduce the intensity and severity of Asthma attacks among children in Algeria}

\author{
Delimi Omar, Zabchi Nor Eddine* and Mkrani Djamel \\ Physical Education and Sport Activities Programmes Evaluation Laboratory, Physical Education \\ and Sports Institute, University Mostganem, Algeria
}

\section{Abstract}

Asthma is one of the most common chronic diseases in the world and affects people of all ages. But having an asthma patient with a good overall fitness level helps reduce the chances of getting a seizure. Aqua-aerobic exercises is appropriate programs for the treatment of asthma. The aim of this study was to propose a rehabilitation program using aqua-aerobic exercises and to determine its effect on some functional parameters (forced Expiratory Volume in first Second Fev1, Peak Expiratory Flow PEF, and the Forced Vital Capacity FVC), and improve the respiratory functions to reduce the intensity and severity of asthma attacks for asthmatic children. This program was applied to a sample of 09 children with light to severe asthma and who are involved in the Asthma Association in Mostaganem- Algeria. The application period lasted 20 weeks, with three training units per week. We relied on the experimental approach and used one group as a sample. Moreover, we adopted pre and post measurements in addition to interstitial measurements at the end of each month using a Spirolab device. The results showed statistically significant differences between the pre and post measurement in the values of respiratory indicators, in favor of the latter. This proves that aqua-aerobic exercises improve the function and efficiency of the respiratory system, and thus reduce the severity of asthma attacks for asthmatic children.

\section{Introduction}

Asthma is a chronic inflammatory disorder in the air paths, accompanied by hyperneuropathy, leading to repeated episodes of cough, muscle, respiratory distress and pressure on the chest [1], where the lungs become infected and the ducts that carry air from and to the lung, and thus causing difficulty to breathe. The air paths of an asthmatic person are very sensitive to certain factors called irritants. When stimulated by irritants, the inflows inflame, swell, increase their secretion to the mucus and contract their muscles, thereby obstructing the normal flow of air [2].

As defined in the clinical medical dictionary, it is a recurrent respiratory disease characterized by an overstatement response due to a variety of aerobic pulmonary embolism, translated into obstructive phenomena of the lower air paths (Belair, 1974, p 253). About 532 million people are infected all over the world [3]. It is one of the most common diseases affecting people of all ages, and is the most common chronic disease (long term), covering twice the number of adults and six times of children, 
with one child out of 8 children suffering from it. Doctors receive more than 18,000 consultations per week, and hospitals count about 74,000 emergency cases every year, and so is the case for all European countries [4].

As for Algeria, over the past 20 to 30 years, respiratory diseases and allergic diseases have increased dramatically, with an estimated number of $8.7 \%$ of patients with asthma [5]. It has become increasingly common in non-developed countries, where inadequate diagnosis and treatment are common, effective medicines may not available or affordable, and it considered as one of the most common causes of hospitalization, especially among children [6].

In this regard, some studies have shown that good physical fitness helps asthma patients reduce the chances of respiratory crises that are due to physical exertion, as physical activity contributes to the discharge of mucous discharge of the lung better, and also increases the depth of breathing, which improves the patients' condition and increases their well-being. So it is possible for patients to exercise all types of sports provided that the increase of the loads is gradual, and make of the physical activity a therapeutic supplement prescribed by doctors in various areas of disease, especially respiratory patients.

Among the recommended practices for asthmatic people are exercises in the aquatic environment where no swimming skills are required, these exercises can be performed in deep and non-deep basins using certain means and tools for fixing and carrying the body to take floating positions. Aqua-aerobic programs are programs that have started to be used recently, and became a new widespread pattern in the programs of physical activity for their positive effects on the physiological, physical and psychological side of patients. They depend on fat as a source of energy for the production of air [7]. Water activities are becoming more and more common in modern societies due to the availability of facilities and services to perform various activities, in addition to the availability of qualified personnel to supervise these water activities and to follow up the situation of practitioners. These aerobic exercises, such as aquaaerobics, burn more calories, lower blood cholesterol, and boost the strength of the respiratory muscles [8].

If we come to observe aquatic exercises we remark that they are very similar to aerobic exercises, the latter have been modified to aqua-aerobic exercises for their effectiveness and health benefits to patients, such as increasing lung volume, improving breathing and overall fitness of patients [9]. Schill notes that the lack of physical activity is due to respiratory fatigue or fatigue of the diaphragm in particular, where it is ascertained that the performance of respiratory functions depends largely on diaphragm functions [10]. Diving in the water and neck dipping help the breathing process up to $60 \%$. This is due to two main factors: pressure on the chest, and high blood pressure in the body. Therefore, these two factors help in the development of breathing muscles leading to good breathing (Kasee Hildenbrand, 2010, p 28).

Several studies, including the study of Abeer Abdel Rahman and Samar Abdel Aziz (1999), concluded that aqua-aerobic exercise improves the efficiency of respiratory and circulatory systems and decreases weight and psychological pressure in patients with some chronic diseases.

In addition, the study of k. Betata et al. (2009) which was carried out for one year on 13 school children with partial or uncontrolled asthma, following a treatment program, within which the researchers conducted a set of measurements by L'ACT and PEAK-FLOW before and after each training session, it was noted that there was a remarkable development in many indicators at varying degrees: PEF, 53\%, Fev1 with $30 \%$, and $46 \%$ for FVC. It was remarked that $8 \%$ of the children's asthma became controllable and that swimming is beneficial for asthmatic children, as it contributes to 
the improvement of respiratory functions and daily life. Among other researches that dealt with the topic of our research, the one of Beggs 2013, it showed that simple and complex aqua-aerobic exercises improve breathing for asthmatic people and reduce the severity of asthma attacks, especially in the case of children.

In the light of all these data, we proposed a habilitation program that includes aqua-aerobic exercises and applied to asthmatic children in order to determine the level of the impact of this program on the respiratory system, and precisely the work of the muscles of the diaphragm, the ability to perform good exhalation and improve breathing functions, and develop the values of respiratory indicators, in addition to the extent of its impact on the severity of asthma for children.

The problematic of the research lies in the two following questions is:

1. Does aqua-aerobic exercise have a role in the development of the respiratory muscles, especially the diaphragm, and thus, in increasing the efficiency of the respiratory system?

2. Does aqua-aerobic exercise have a positive effect on the respiratory system and improve some respiratory indicators to reduce the severity of asthma attacks for children?

\section{Method and Tools}

The nature of our study required the use the experimental method. We designed the sample group in addition to pre and post measurements. The sample of the study included (09) children from asthmatic patients, with different degrees of infection, ranging from light to severe. As for the sample's age, it ranges from 07 to 12 years, $18 \%$ of them were enrolled in the Asthma Association in Mostaganem- Algeria, i.e. $(50 \%)$ of the total number. The study took a period of five months after parental authorization allowed them to swim. The sample was subjected to aqua-aerobic exercises in the period between the pre and post measurements, it took place in the swimming pool of Sport and Physical Education Institute of Mostaganem University. For the measurement we adopted the spirobankП tool to measure some important respiratory indicators which are used by specialists to diagnose and know the severity of the disease in an objective way, and to know the strength of the breathing muscles: (forced expiratory volume in one second FEV1), Peak Expiratory Flow (PEF), and the Forced Vital Capacity (FVC). We have also adopted criteria that determine the levels of asthma severity (Severe Persistent, Moderate Persistent, Intermittent and mild persistent), which is the division of the Global Initiative of Asthma (GINA). In order to analyze the data we used the statistical program [11].

\section{Results}

\section{Pre-test and post-test results for FVC}

According to table 1 that shows the results of the FVC according to severity, there is an improvement in the FVC after the application of the rehabilitation program using aqua-aerobic exercises, regarding the differences between the frequencies and percentages of the pre and post measurements according to the defined levels of severity of asthma. The pre measurement showed that most of the children of the sample suffer a severe continuous asthma with a rate of (44.4\%), followed by $33.3 \%$ of children Intermittent and mild persistent, and a small group $(22.2 \%)$ with an Intermittent and mild persistent, while the post measurement showed that the majority of the children in question (66.6\%) became at the level of Intermittent and mild persistent asthma (Figure 1).

According to the results shown in table 2, which shows the t-test calculated values of the first and second dimensions forced vital capacity (FVC), which were estimated 
at2.784- at the significance level of 0.05 and the freedom level of 8 , and the incorporeal value of 0.02 , indicating statistical differences in the pre and post measurement of forced vital capacity (FVC).

According to table 3 that shows the results of pre and post measurements of the (forced expiratory volume in one second (FEV1) according to severity, there is an improvement in the volume of expiratory volume in one second (FEV1) after application of the rehabilitation program using aqua-aerobic exercises. The results of the pre measurement showed that the majority of the children were suffering from severe or moderate asthma, while the results of the post measurement showed that the majority of the children in question (66.6\%) became at the level Intermittent and mild persistent asthma (Figure 2).

According to the results shown in table 4, which shows the t-test calculated values of the first and second dimensions of forced expiratory volume in one second (FEV1), which were estimated at_ 2.625 at the significance level of 0.05 and the freedom level of 8 , and the incorporeal value of 0.03 , indicating statistical differences in the pre and post measurement of forced expiratory volume in one second (FEV1).

\section{Results of the pre and post peak expiratory flow (PEF)}

In table 5, which shows the results of the pre peak and postoperative maximum runoff (PEF) according to severity, there appears to be a slight improvement in maximum exhalation (PEF) after application of the rehabilitation program using aquaaerobic exercises. This improvement appears through the differences that emerged between the frequency and percentages of the pre and post measurements according to the levels of asthma severity, where the results showed that two of the three children with severe asthma improved and became at the level of moderate asthma (Figure 3).

The results shown in table 6 indicate the calculated value $\mathrm{T}$ of the pre and post measurements of the Maximum Exhaustion Flow (PEF) which was estimated at 1.705 at the significance level of 0.05 and the freedom degree of 8 , and reached the incorporeal value of 0.12 which is greater than 0.05 , indicating that there no statistically significant differences in the pre and post measurements of the maximum expiratory flow (PEF).

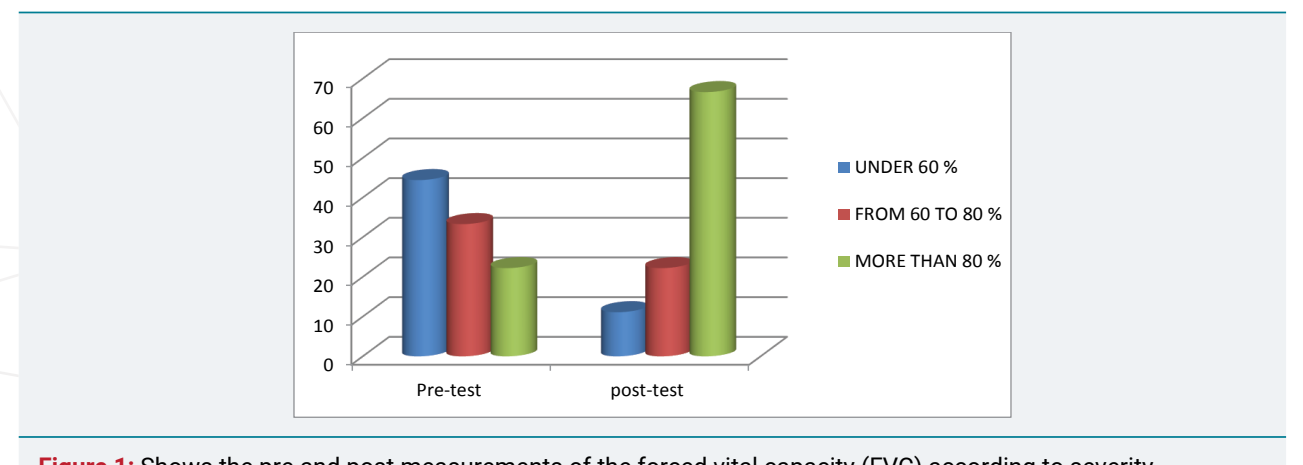

Figure 1: Shows the pre and post measurements of the forced vital capacity (FVC) according to severity.

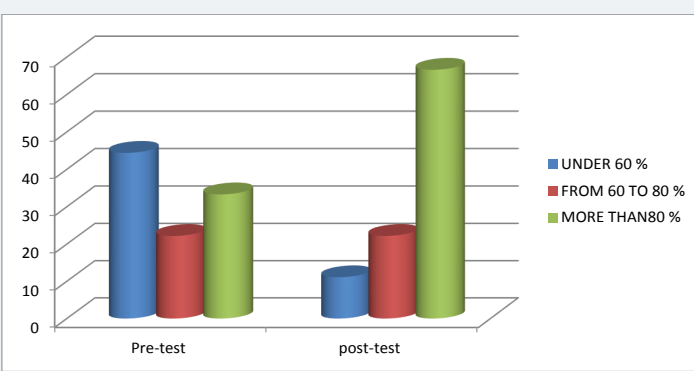

Figure 2: The pre measurement of the forced expiratory volume in one second (FEV1) according to severity. 


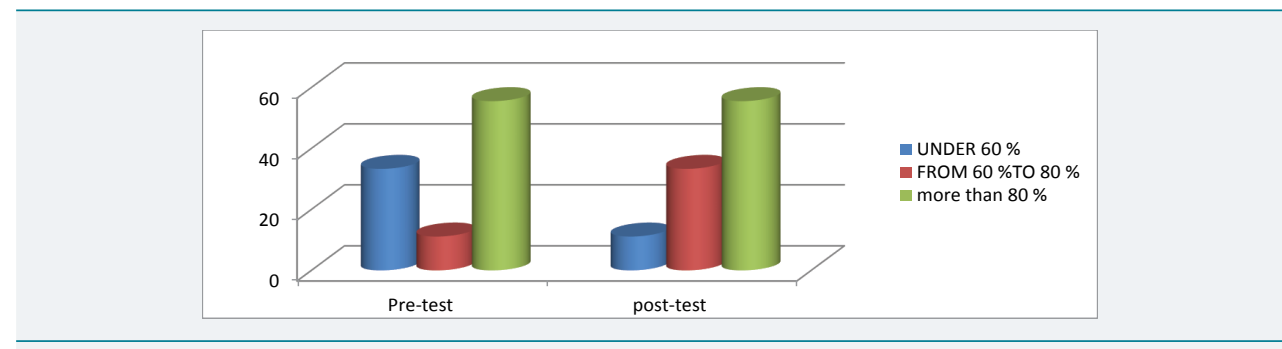

Figure 3: Shows the pre and post measurements of Peak expiratory flow (PEF) according to severity.

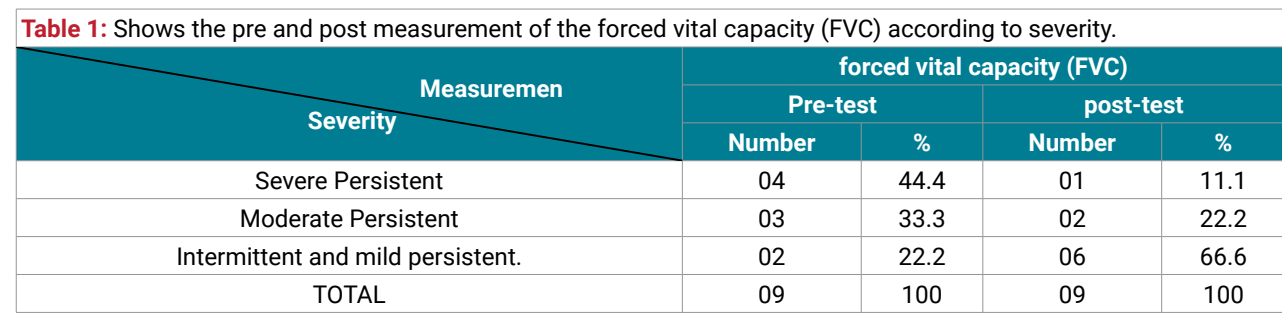

Table 2: Shows the results of t-test for the forced vital capacity (FVC) for the pre and post measurements.

\begin{tabular}{|c|c|c|c|c|c|c|}
\hline measurement & M & SD & DF & t-test & significance & SIG \\
\hline Pre-test & 59.55 & 20.20 & 08 & $2.784-$ & sig & 0.02 \\
\hline post-test & 80.66 & 14.64 & & & & \\
\hline
\end{tabular}

Table 3: The pre and post measurement of the forced expiratory volume in one second (FEV11) according to severity.

\begin{tabular}{|c|c|c|c|c|}
\hline Measurement & \multicolumn{3}{c|}{ Forced expiratory volume in the first second FEV1) } \\
\hline & \multicolumn{2}{|c|}{ Pre-test } & \multicolumn{2}{c|}{ post-test } \\
\hline & Number & $\%$ & Number & $\%$ \\
\hline Severe Persistent & 04 & 44.4 & 01 & 11.1 \\
\hline Moderate Persistent & 03 & 22.2 & 02 & 22.2 \\
\hline Intermittent and mild persistent. & 02 & 33.3 & 06 & 66.6 \\
\hline TOTAL & 09 & 100 & 09 & 100 \\
\hline
\end{tabular}

Table 4: Shows the results of t-test for the forced expiratory volume in one second (FEV1) for the pre and post measurements.

\begin{tabular}{|c|c|c|c|c|c|c|}
\hline Measurement & M & SD & DF & t-test & significance & SIG \\
\hline Pre-test & 67.11 & 22.21 & 08 & $-2.625-$ & sig & 0.03 \\
\hline post-test & 89.33 & 16.75 & & & & \\
\hline
\end{tabular}

Table 5: Shows the pre and post measurements of Peak expiratory flow (PEF) according to severity.

\begin{tabular}{|c|c|c|c|c|}
\hline Measurement & \multicolumn{3}{c|}{ Peak expiratory flow (PEF) } \\
& \multicolumn{2}{|c|}{ Pre-test } & \multicolumn{2}{c|}{ post-test } \\
\hline Severe Persistent & Number & $\%$ & Number & $\%$ \\
\hline Moderate Persistent & 03 & 33.3 & 01 & 11.1 \\
\hline Intermittent and mild persistent. & 01 & 11.1 & 03 & 33.3 \\
\hline TOTAL & 05 & 55.5 & 05 & 55.5 \\
\hline
\end{tabular}

Table 6: Shows the results of t-test for the forced expiratory volume in one second (FEV1) for the pre and post measurements.

\begin{tabular}{|c|c|c|c|c|c|c|}
\hline Measurement & M & SD & DF & t-test & Significance & SIG \\
\hline Pre-test & 76.00 & 22.54 & 08 & $-1.705-$ & sig & 0.12 \\
\hline post-test & 76.00 & 22.54 & & & & \\
\hline
\end{tabular}

\section{Discussion}

The improvement of the FVC in all study subjects indicates that the applied aerobic exercise program has had an effect. This indicator shows the goodness and strength of the respiratory system and the improvement of the respiratory muscles of the children concerned by the research, as most of them experienced a decrease in the severity of asthma, as it became controllable, and this has improved their lives. These results 
go in line with the studies of Hildenbrand (2002) and Sheel (2010), as they confirm that the performance of respiratory functions depends largely on the functions of the diaphragm, and that aqua-aerobic activities in general help in the development of respiratory muscles leading to good breathing. Moreover the study of (K. Bentta, .N. Lekhal, B.Ziane, Y.Berrabah, 2009) and the study of (Beggs, 2013) proved that simple and complex aqua-aerobic exercises may improve breathing for people with asthma and reduce the severity of asthma attacks for children.

In addition, the vasomotor volume index (FEV1) was improved due to the effect of aqua- aerobic exercises on the competence of the respiratory system, especially the respiratory muscles (muscles between ribs and diaphragm). This is confirmed by the results of the study of Abeer Abdul Rahman and Sahar Abdul Aziz, which pointed out that regular aqua exercise improve the functions of the lungs and improve the efficiency of the periodic system, as it reduces stress and helps in weight loss. In addition, the study of Wadell, K. (Wadell, $\mathrm{K}$ et al, 2005) and the study Kurabayashi (Kurabayashi H et al. 2000), showed that the gradual exercise in water caused changes in walk distances as proved through walking test, as it improved respiratory functions, especially FVC and FEV1.

As for the maximum exhalation flow (PEF) the results were not significant, because the use of this indicator for several times was for the safety of children and asthma control, since its value indicates the state of asthma in the patient on the one hand and on the other to avoid crises during the training. According to the classifications of the Global Initiative for Asthma (GINA) and the National Program for Education and Prevention from Asthma (NAEPP), the value of the PEF index of those classified with severity (sharp and light) is greater than $80 \%$ that is why no improvement was observed with this category. These results are different from those of K.Bennetta, N.Lekhal, B.Ziane, and Y. Berrabah (2009).

\section{Conclusion}

Aqua-aerobic exercise has a positive effect on the physiological variables, especially the pulmonary functions of asthmatic patients (FVC and FEV1). The aerobic exercise also has an effective role in improving respiratory efficiency, specifically developing cardiovascular abilities, improving aerobic abilities, increasing the depth of breathing and improving muscle strength, Respiratory muscles, which reduced the intensity and severity of asthma in children. Children with asthma can exercise physically, provided there is some kind of regulation and monitoring, that is to say a gradual increase in the intensity of exercise, and it is preferable to apply the Borg scale. All of this improves the lives of children suffering from Asthma.

\section{References}

1. Global Initiative for Asthma. Global strategy for asthma management and prevention. 2016;

2. Swinkey I. Chronic respiratory diseases. 2017; Ref.: https://goo.gl/t7aom8

3. America AA. Consulté le juin 02, 2017; Ref.: https://goo.gl/ed5Wby

4. Yongs R. How to coexist with asthma. Cairo: Dar El Farouk For Publication and distribution, First Edition. 2015;

5. Anane BE. Guide sue le diagnostic et la prise en charge de l'asthme de l'enfant. algérie: société algérienne de pédiatrie. 2010; Ref.:

6. Akinbami LJ, Houma DM, Manino DM. Control of Asthma. The United States of America: summary of Morbidity and Mortality Weekly Report. 2002;

7. Arabi SM, ElDomeiri HH. The impact of the water exercises on some physical and physiological variables and blood sugar level to infected with type 2 diabetes in Jordan. (University of Jordan, the editor) Studies of Educational Sciences, First Edition. 
8. Bhaskarabhtla KV. Physical activity and type 2. Diabetes physician and sports medecine. 2004; 32: 13-17. Ref.: https://goo.gl/eYJNpi

9. Gelinas JE, Reid G. The developmental validity of traditional learn to swin progressions for children with physical disabilities. canada: national library. 2000; 17: 269-285. Ref.: https://goo.gl/jtfQ71

10. Sheel AW, Derchak PA, Pegelow DF, Dempsey JA. Threshold effects of respiratory muscle work on limb vascular resistance. Am J Physiol Heart Circ Physiol. 2002; 282: H1732-1738. Ref.: https://goo.gl/x4806c

11. Connel RM, Jones. Improuvedathletic performance in the generale population. dictionnair emedical clinique pharmacologie et therapeutique-ed-5.

12. Hilmi I, Farhat LA. Physical Education and Recreation for the handicapped. Cairo: Dar El Fikre Al Arabi. 1998;

13. Djaoudet M. Statistical analysis using (SPSS(, 298. Amman - Jordan: Dar Wael for publication and distribution. ) - First Edition. 